\section{SOCIAL INTERVENTION IN THE FAMILIES OF SCHIZOPHRENICS: ADDENDUM}

DeAR Sir,

In the journal (August, 1982, 141, 121-34) we reported the results of a trial of social intervention in the families of schizophrenic patients. We presented data on 23 of the 24 patients who entered the trial, as the final patient remained a year in hospital before being discharged, so that waiting for her follow-up would have delayed considerably the presentation of our findings. The ninth-month follow-up of this patient has now been carried out, and we present the results here for the sake of completeness.

The patient was aged 41 at the key admission and was a single woman who had lived with her mother all her life. She had first been admitted for schizophrenia five years earlier and had recovered after several weeks hospitalisation. She returned to her job as a clerk and remained well until shortly before her second admission, although her work efficiency was somewhat impaired. On admission she spoke very little, but did complain spontaneously of voices from all around her that sometimes spoke about her. A few days later she became completely mute and remained so for some months despite active treatment. Her clinical state fluctuated considerably over the course of her admission and she appeared to be at risk of becoming institutionalised. Almost a year after admission she was discharged home to her mother, free from florid symptoms, but with blunting of affect and no spontaneous speech.

Her mother was assessed for expressed emotion (EE) initially and produced 27 critical comments, and was scored 1 on hostility and 0 on overinvolvment. The patient spent all her leisure time with her mother, and clocked up $47 \frac{1}{2}$ hours of face-to-face contact per week. Random assignment resulted in this family joining the experimental group. The mother was given the education programme and then attended the relatives' group regularly. However the group terminated several months afterwards, following which a research psychologist saw the couple jointly in their home once a fortnight for the rest of the follow-up period.

The patient was too handicapped to resume work, and instead attended a day hospital three times a week, alternating with a day centre twice a week. Despite this she remained in high contact with her mother (391 hours per week) at follow-up. At the nine-month assessment, mother's EE was rated by two independent assessors as: critical comments 3 , hostility 0 , overinvolvment 2 . Thus she had changed from high to low EE. The patient was maintained on long-acting injections and experienced no recurrence of florid symptoms during the nine-month follow-up period. Her affect remained rather restricted, but she showed much more spontaneous speech, had resumed some hobbies, such as knitting, and helped with chores, in particular keeping her own room clean and tidy.

When this mother's scores on criticism are added to the previous data for experimental relatives, the mean number of critical comments falls from 16.7 to 6.5 following intervention $(t=3.7$, df, $11, P<0.005)$. Including this case, we were successful in achieving one or both of our therapeutic aims in nine out of the 12 families ( 75 per cent). The relapse rate in the control group was six out of 12 ( 50 per cent), while the rate in the whole experimental group was one out of 12 (eight per cent, exact $P=0.032$ ). In the nine families in which the social intervention achieved its aims, not a single patient relapsed, a difference from the relapse rate in the control group of even greater significance (exact $P=0.017$ ).

The follow-up of this last patient has increased the differences between the experimental and the control group and has thus strengthened the evidence both for the causal role of relatives' $E E$ in schizophrenic relapse and for the therapeutic effectiveness of working with these families.

JULIAN LEFF

LIZ KUIPERS

RUTH BERKOWITZ

MRC Social Psychiatry Unit, Institute of Psychiatry, Denmark Hill, London, SE5 8AF

ROSEMARIE EBERLEIN FrIES
Psychiatrische Universitaetsklinik, Munich

DAVID StURGEON

Department of Mental Health, University College, London

\section{HYSTERECTOMY FOR MENORRHAGIA}

DEAR SIR,

I notice that most of the papers and letters disapproving of hysterectomy for functional menorrhagia emanate from men who, ipso facto, can have no first-hand experience of the problems. Writing as one who has the somewhat double-edged advantage of personal experience of menorrhagia, I can assure these gentlemen that having prolonged and/or heavy periods-(particularly if they are also somewhat irregular) - can play havoc with one's budget, one's social and sporting activities, and packing, and result in spoilt holidays, not to mention spoilt clothing and bedding, plus a lot of embarrassment. It is hardly surprising if women who have suffered in this way for some years develop psycho-social disturbances. P. J. H. GosLing

Mount Pleasant Hospital, Frederick Road, Ore, Hastings TN35 5AA 